\title{
Electrical stimulation of the cochlea
} for treatment of chronic disabling tinnitus: an open-label trial towards the development of an implantable device

\author{
John P. Marinelli1,2 , C. Lane Anzalone ${ }^{1}$, Christoph M. Prummer ${ }^{1}$, Gayla L. Poling ${ }^{1}$, Jeffrey P. Staab3
}

Nicole M. Tombers ${ }^{1}$, Christine M. Lohse ${ }^{4}$ and Matthew L. Carlson ${ }^{1,5^{*}}$

\begin{abstract}
Background: Chronic tinnitus affects millions of people globally and constitutes the most commonly compensated disability among military service members in the United States. Existing treatment options largely surround helping patients cope with their disease as opposed to directly suppressing tinnitus perception. The current study investigated the efficacy of electrical stimulation of the cochlea on chronic disabling tinnitus.

Methods: In this single-arm, open-label clinical trial, 22 adult subjects with severe-range asymmetric or unilateral non-pulsatile tinnitus underwent electrical stimulation of the cochlea through use of an extra-cochlear electrode positioned on the cochlear promontory. Each subject underwent 3 stimulation treatments over 3 weeks at 7-day intervals. Tinnitus severity was determined by Tinnitus Handicap Inventory (THI), Tinnitus Functional Index (TFI), and Tinnitus Visual Analog Scale (VAS). Inclusion criteria required subjects have no worse than moderate sensorineural hearing loss determined by pre-enrollment audiometric testing. The primary outcome was nadir post-treatment THI scores, obtained at seven timepoints following electrical stimulation, with clinically significant improvement defined as a decrease of $\geq 7$.
\end{abstract}

Results: All 22 (100\%) subjects experienced clinically significant improvement in the THI during the study period with a mean decrease in scores of $-31(95 \% \mathrm{Cl}-38$ to -25$)$ from a baseline of 48 . Twenty (91\%) experienced clinically significant improvement detectable on at least two of the three tinnitus survey instruments and 17 (77\%) experienced clinically significant improvement detectable on all three survey instruments (i.e., THI, TFI, and VAS). Eight (36\%) subjects reported either complete (THI of $0 ; n=3$ ) or near-complete (THI $1-4 ; n=5)$ suppression of their tinnitus following a stimulation session. Thirteen (59\%) subjects reported a nadir following stimulation at or below the threshold for "no or slight handicap" on the THI $(\leq 16)$. No adverse events were observed.

Conclusions: These findings establish the foundation for the development of an extra-cochlear implantable device that delivers electrical stimulation to the cochlea for the treatment of disabling tinnitus. For patients considering device implantation, trans-tympanic cochlear promontory stimulation can facilitate patient selection.

Trial Registration ClinicalTrials.gov Identifier: NCT03759834. URL: https:/clinicaltrials.gov/ct2/show/NCT03759834

*Correspondence: carlson.matthew@mayo.edu

1 Department of Otolaryngology-Head and Neck Surgery, Mayo Clinic,

Rochester, MN 200 1st St SW55905, USA

Full list of author information is available at the end of the article

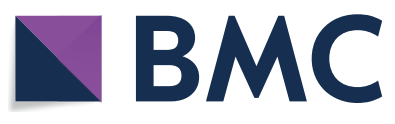

(c) The Author(s) 2022. Open Access This article is licensed under a Creative Commons Attribution 4.0 International License, which permits use, sharing, adaptation, distribution and reproduction in any medium or format, as long as you give appropriate credit to the original author(s) and the source, provide a link to the Creative Commons licence, and indicate if changes were made. The images or other third party material in this article are included in the article's Creative Commons licence, unless indicated otherwise in a credit line to the material. If material is not included in the article's Creative Commons licence and your intended use is not permitted by statutory regulation or exceeds the permitted use, you will need to obtain permission directly from the copyright holder. To view a copy of this licence, visit http://creativecommons.org/licenses/by/4.0/. The Creative Commons Public Domain Dedication waiver (http://creativeco mmons.org/publicdomain/zero/1.0/) applies to the data made available in this article, unless otherwise stated in a credit line to the data. 
Keywords: Tinnitus, Treatment, Electrical stimulation, Promontory stimulation, Implantable device, Cochlear implant, Clinical trial

\section{Background}

In the absence of approved pharmacological or surgical therapies, millions of people live with disabling chronic tinnitus [1-3]. It is estimated that 2 million residents of the United States are severely impacted by chronic tinnitus, and tinnitus constitutes the most commonly compensated service-connected disability among veterans nationally [1-3]. Although the etiology of tinnitus is complex and multifactorial, [3] growing evidence surrounding tinnitus outcomes among patients undergoing cochlear implantation for moderate to profound sensorineural hearing loss has demonstrated that almost 90\% of patients with preoperative tinnitus experience notable improvement and up to $45 \%$ experience complete suppression of their tinnitus during device use [4-7].

Analogous to the nerve stimulation treatment of neuropathic pain in peripheral nerve disorders, the therapeutic effect of cochlear implantation on chronic tinnitus appears to be related to electrical stimulation of the cochlea and independent of auditory masking [8]. Moreover, the observed therapeutic effect has been shown to be durable up to at least 10 years [9]. Yet, because cochlear implantation places native hearing at risk, it is contraindicated in patients with functional natural hearing. However, most patients with disabling chronic tinnitus have no worse than moderate hearing loss $[1,3]$. Attempting to replicate the electrical stimulation of the cochlea during cochlear implant device use, an open label clinical trial was undertaken to evaluate the utility of trans-tympanic electrical stimulation of the cochlear promontory in adults who suffer from bothersome chronic unilateral or asymmetric tinnitus but do not have hearing loss of sufficient severity to qualify for cochlear implantation.

\section{Methods}

After obtaining institutional review board approval, the protocol was registered with ClinicalTrials.gov (NCT03759834). Enrollment criteria stipulated that subjects were $\geq 18$ years old with no worse than moderate sensorineural hearing loss in the study ear (based on a pure-tone average of hearing thresholds at 500, $1000,2000 \mathrm{~Hz}$, and $3000 \mathrm{~Hz}$ of $<70 \mathrm{~dB}$ on pre-enrollment audiometric testing). Word recognition scores had to be $>75 \%$. Inclusion criteria required subjects undergo pre-enrollment neuroimaging with both gadolinium-enhanced magnetic resonance imaging of the head and high-resolution computed tomography of the temporal bones, and both studies had to be read as normal for subject age by a neuroradiologist from our institution.

Subjects' tinnitus had to be non-pulsatile, unilateral or definitively asymmetric by subject report, and be present for at least 6 months but less than 3 years. Subjects' tinnitus had to be disruptive, determined by subject reporting "severe-range" tinnitus on the Tinnitus Handicap Inventory (THI), [10, 11] Tinnitus Functional Index (TFI), [12] or Tinnitus Visual Analog Scale (VAS) [13]. The tinnitus also had to be intractable and fail conventional methods of therapy such as auditory masking with hearing aid use.

Exclusion criteria excluded subjects who were pregnant, had a history of brain or lateral skull base surgery, had a history of major head trauma, and those actively using any tinnitus treatments outside of masking devices (e.g., noise generators, hearing aids). Subjects could not be taking antidepressants, anxiolytics, or antipsychotics and could not have clinically significant anxiety (determined by a pre-enrollment Generalized Anxiety Disorder-7 score >9), clinically significant depression (determined by a pre-enrollment Patient Health Questionnaire-8 score $>9$ ), or hypochondriacal level illness anxiety (determined by a pre-enrollment Short Health Anxiety Inventory score $>25$ ). Subjects received no financial compensation for participation. The study was performed between December 2017 and September 2018.

Each subject underwent 3 stimulation treatments over 3 weeks at 7 -day intervals. Promontory stimulation was performed using biphasic charge balanced pulses (Cochlear Nucleus Promontory Stimulator Z10012 ${ }^{\circledR}$, Cochlear Corporation, Melbourne, Australia; Fig. 1). Following trans-tympanic placement of an insulated monopolar stimulation probe, electrode impedance testing was performed to evaluate proper electrode placement. Gentle repositioning of the electrode or replacement through the tympanic membrane was performed when impedance values were not acceptable. Next, calibration testing to assess optimal stimulation parameters for each therapeutic session was carried out using an output of $0-1000 \mu \mathrm{A}$ for pulse frequencies 100, 800, and $1600 \mathrm{~Hz}$ (Additional file 1: Table S1). As an internal control function, testing included a run of "on-off" stimulations blinded to the subject (i.e., subject was blinded to the status of the "on" or "off" position of the stimulation machine) to document subject 


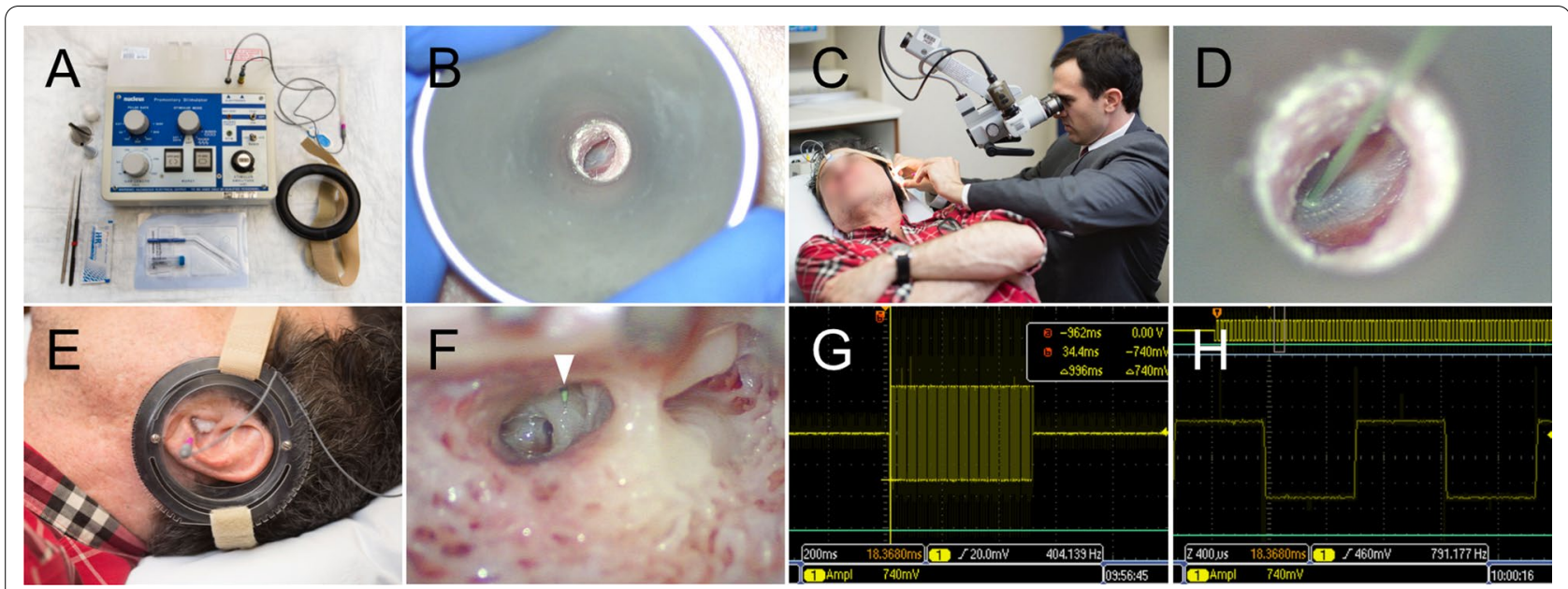

Fig. 1 Trans-tympanic electrical stimulation of the cochlea. A Equipment used for promontory stimulation, including the Cochlear Nucleus Promontory Stimulator Z10012 ${ }^{\circledR}$ (Cochlear Corporation, Melbourne, Australia). B Otomicroscopic view of the left tympanic membrane. C, D Surgeon placing the trans-tympanic monopolar needle electrode on the promontory. $\mathbf{E}$ Stabilized electrode in left ear. $\mathbf{F}$ Typical location of the needle electrode on the promontory of the cochlea is shown (white arrowhead) through a facial-recess approach commonly used for cochlear implantation[22]. G, H oscilloscope recording of promontory stimulation output demonstrating charge-balanced square biphasic pulse waveforms

perception of stimulation. Electrical current levels were gradually titrated to determine the maximal comfortable threshold as reported by the subject. The subject then underwent $10 \mathrm{~min}$ of trans-tympanic electrical stimulation at $80 \%$ of the maximum comfort threshold for each of the predetermined pulse frequencies. The primary outcome measure of interest was nadir in THI following electrical stimulation.

Immediately prior to each treatment, comprehensive audiometric testing was performed. To monitor the potential for delayed audiologic complications, physical examination with binocular microscopy and comprehensive audiometric testing was completed at 3 months following the third promontory stimulation treatment. Information regarding the specific tinnitus survey instruments, subject survey reporting schedule, and statistical power estimations can be accessed in the online supplement (Additional file 1: Text S1).

\section{Results}

Twenty-five subjects initially enrolled in the study. Three subjects withdrew prior to undergoing promontory stimulation due to an inability to complete the multiple requirements of the study. Therefore, 22 subjects underwent promontory stimulation (Table 1); 21 (95\%) subjects completed the study, with one subject withdrawing after the first stimulation treatment due to a perceived inability to complete the remaining requirements in the study; their reported data until self-exclusion were included in
Table 1 Baseline clinical features of study cohort, $\mathrm{n}=22$

\begin{tabular}{ll}
\hline Feature $^{\text {a }}$ & \\
\hline Demographics & \\
Age at enrollment in years & $59(8)$ \\
Sex & \\
$\quad$ Female & $8(36)$ \\
$\quad$ Male & $14(64)$ \\
Eligibility criteria & \\
Tinnitus Handicap Inventory & $57(16)$ \\
Tinnitus Functional Index & $70(10)$ \\
Visual Analog Scale & $7.9(1.4)$ \\
Generalized Anxiety Disorder-7 & $1.5(0-3)$ \\
Patient Health Questionnaire-8 & $2.5(1-5)$ \\
Short Health Anxiety Inventory & $7.5(5.6)$ \\
Tinnitus characteristics & \\
Duration of tinnitus in years $(n=19)$ & $2.0(0.8)$ \\
Persistence $(n=20)$ & \\
Constant & $18(90)$ \\
Intermittent & $2(10)$ \\
Fluctuations in intensity or loudness $(n=20)$ & $15(75)$ \\
Treatments $(n=20)$ & \\
Masker & $8(40)$ \\
Hearing aid & $5(25)$ \\
Music therapy & $3(15)$ \\
Cinnitus retraining therapy & $1(5)$ \\
None & $1(5)$ \\
\hline
\end{tabular}

${ }^{\mathrm{a}}$ Summarized with mean (SD), median (IQR), or $\mathrm{n}(\%)$ 
efficacy and safety endpoints. No subjects experienced any adverse events, including no persistent pain or discomfort following stimulation and no subjective change in hearing thresholds. Further, physical exam and comprehensive audiometric testing demonstrated no clinically detectable change from baseline to the 3-month post-treatment testing (Additional file 1: Table S2).

All $22(100 \%)$ subjects experienced clinically significant improvement in the THI (score decrease by $\geq 7$ ) [11] during the study period with a mean decrease in scores of -31 (95\% CI -38 to - 25) from a baseline of 48 (Table 2). Twenty (91\%) experienced clinically significant improvement detectable on at least two of the three tinnitus survey instruments and 17 (77\%) experienced clinically significant improvement detectable on all three survey instruments (i.e., THI, TFI, and VAS). Eight (36\%) subjects reported either complete (THI of $0 ; n=3$ ) or near-complete (THI 1-4; $\mathrm{n}=5$ ) suppression of their tinnitus following a stimulation session. Thirteen (59\%) subjects reported a nadir following stimulation at or below the threshold for "no or slight handicap" on the THI $(\leq 16)$. At 3 months, most subjects had returned close to their baseline tinnitus severity levels, although 4 (18\%) subjects exhibited sustained suppression of $\leq 16$ on the THI.

\section{Discussion}

Cochlear implantation has emerged as an effective treatment for bothersome chronic tinnitus in adults with concomitant moderate to profound sensorineural hearing loss $[5,7]$. The therapeutic effect appears independent of the improved access to sound and has been shown to be durable in long-term follow-up [8, 9]. Preliminary mechanistic evidence suggests that the electrical current levels delivered by most modern cochlear implants may inactivate voltage-gated calcium channels that play a role

Table 2 Tinnitus severity survey reporting during study duration, $\mathrm{n}=22$

\begin{tabular}{|c|c|c|c|c|}
\hline Survey & Baseline $^{a}$ & $\begin{array}{l}\text { Post- } \\
\text { stimulation } \\
\text { Session Nadir }\end{array}$ & Difference $^{a, b}$ & $\begin{array}{l}95 \% \mathrm{Cl} \text { for } \\
\text { difference }\end{array}$ \\
\hline $\mathrm{THI}$ & $48(15)$ & $17(17)$ & $-31(14)$ & -38 to -25 \\
\hline TFI & $60(13)$ & $25(20)$ & $-35(16)$ & -42 to -28 \\
\hline VAS & $7.1(1.4)$ & $3.4(2.5)$ & $-3.7(2.1)$ & -4.7 to -2.8 \\
\hline
\end{tabular}

THI Tinnitus Handicap Inventory; TFI Tinnitus Functional Index; VAS Visual Analog Scale

a Summarized with mean (SD)

${ }^{b}$ Defined as lowest post-stimulation score minus baseline score. Baseline scores were defined as the mean of the enrollment, baseline evaluation 1 , baseline evaluation 2, baseline evaluation 3, and pre-stimulation 1 scores. Post-stimulation session nadir scores were defined as the lowest score reported following a stimulation session in tinnitus generation [14]. Although cochlear implantation is contraindicated in patients with functional natural hearing, the current clinical trial demonstrates that therapeutic electrical stimulation of the cochlea is replicable for temporary periods through trans-tympanic cochlear promontory stimulation.

In the current clinical trial, the mean effect size of subjects' nadir tinnitus level post-treatment exceeded the threshold for clinically significant decreases on the THI by over four-fold. This finding is notable because study subjects' baseline THI is similar to a large proportion of patients suffering from chronic severe tinnitus, and the THI has been widely validated across multiple international studies [11, 15-19]. The clinical significance of the treatment response observed in the present work is also strengthened by a United States survey of 439 civilians and 269 military service members where nearly $75 \%$ of respondents reported being willing to receive an implantable device if it reduced tinnitus by even 50\% [20]. Of note, the rate of complete or near-complete suppression of subjects' tinnitus following trans-tympanic promontory stimulation (approximately $40 \%$ ) is comparable to prior rates observed in the setting of cochlear implantation [7]. Notwithstanding the improvement in tinnitus severity among all study subjects, the duration of tinnitus suppression observed following trans-tympanic cochlear promontory stimulation was limited and returned close to baseline by 3 months following the last stimulation session for most subjects.

The notable but temporary tinnitus suppression effect of trans-tympanic electrical stimulation of the cochlea establishes a foundation for the development of an implantable device for treatment of disabling chronic tinnitus. An implantable device that does not place native hearing at risk would enable patients with functional natural hearing to potentially experience suppression of tinnitus on a daily basis similar to cochlear implant recipients. In this way, an implantable device would not be limited to brief stimulation sessions, as in transtympanic promontory stimulation, but would provide extended durations of tinnitus suppression based on current battery life capabilities of modern cochlear implant technology. Moreover, because one of the most common complaints among those suffering from tinnitus is lack of control of symptoms, a device that affords patients the ability to turn on/off the therapeutic benefit would likely confer unique psychological benefit in a patient population that often suffers from concomitant anxiety and depression $[1,12]$.

Important when considering an implantable device, several pieces of evidence suggest that it is unlikely that long-term electrical stimulation of the cochlea by an extra-cochlear electrode that does not violate the 
cochlear lumen would negatively influence patients' baseline hearing. One of the strongest supports for this extends from the cochlear implant literature. For instance, Roland et al. evaluated long-term audiometric outcomes for patients with high-frequency sensorineural hearing loss who underwent cochlear implantation with the Nucleus ${ }^{\circledR}$ HybridTM L24 (Cochlear Ltd., Sydney, Australia) cochlear implant and had at least 5 years of post-implantation follow-up [21]. The authors found that hearing changes from preoperative to the early postoperative timeframe were significant, but changes 6 months through 5 years post-activation of cochlear stimulation with a cochlear implant were not statistically significantly different [21]. They, along with other similar studies [22], demonstrated that the risk of sensorineural hearing loss following cochlear implantation is due to the upfront risk of violating the cochlear lumen, but the risk of progressive hearing loss after surgery mirrors the slow decline in hearing seen in the opposite, non-implanted ear. In other words, isolated chronic electrical stimulation of the cochlea does not appear to accelerate hearing loss compared to the non-stimulated ear. Similar durable hearing thresholds following long-term electrical stimulation of the cochlea using extra-cochlear electrodes have been demonstrated in animal models [23, 24]. Taken together, there is good reason to believe that extra-cochlear electrical stimulation of the cochlea in humans is unlikely to be harmful to hearing thresholds long-term.

Because the etiology of tinnitus is broad and response to therapy-to include cochlear implantation-varies among patients, patient selection for an extra-cochlear implantable device can be facilitated by a pre-implantation trial of trans-tympanic cochlear promontory stimulation. Twelve subjects who experienced clinically significant improvement across all three tinnitus survey instruments in the current study self-identified as desiring to undergo device implantation with an extracochlear implantable device based on existing cochlear implant technology that is currently under investigation (ClinicalTrials.gov number NCT03988699). Taken together, the current study demonstrates the feasibility and need for an implantable device that replicates the electrical stimulation of the cochlea during cochlear implant device use but does not risk patients' native hearing.

\section{Conclusions}

Electrical stimulation of the cochlea through a transtympanically placed electrode can suppress tinnitus. These results support the possibility that an extra-cochlear implantable device to deliver electrical stimulation to the cochlea can replicate the tinnitus suppression benefits of cochlear implants for most patients with chronic tinnitus who do not have hearing loss of sufficient severity to qualify for cochlear implantation. Given the results of the present study, trans-tympanic cochlear promontory stimulation may facilitate patient selection for device implantation.

\section{Abbreviations \\ THI:Tinnitus Handicap Inventory; TFI: Tinnitus Functional Index; VAS: Tinnitus Visual Analog Scale.}

\section{Supplementary Information}

The online version contains supplementary material available at https://doi. org/10.1186/s12967-022-03271-4.

Additional file 1: Table S1. Summary of promontory stimulation parameters, $\mathrm{n}=22$. Table $\mathbf{S 2}$. Safety data from behavioral audiometric testing across study duration, $\mathrm{n}=22$. Text $\mathbf{S 1}$. Supplementary methods of tinnitus survey information and reporting schedule and statistical power.

Acknowledgements

Not applicable.

\section{Authors' contributions}

MLC provided the primary inspiration for the conception and design of the current work. All authors were instrumental in the data collection, design, and implementation. All authors contributed to the drafting and approval of the final manuscript. All authors take responsibility for all aspects of the current work. All authors read and approved the final manuscript.

\section{Funding}

This work was supported, in part, by the Department of Defense Peer Reviewed Medical Research Program for the Office of the Congressionally Directed Medical Research Programs under award number W81XWH-19-1-0021. Opinions, interpretations, conclusions, and recommendations are those of the authors and are not necessarily endorsed by the Department of Defense.

Availability of data and materials Not applicable.

\section{Declarations}

Ethics approval and consent to participate Mayo Clinic IRB 17-004832.

\section{Consent for publication}

Not applicable.

\section{Competing interests}

M.L.C. reports holding pending patents 62/346,306, PCT/US2017/035617, and WO2017213978A1 on devices and methods for treating tinnitus with the use of electrical stimulation. No other authors have relevant conflicts of interest to disclose. The views expressed herein are those of the authors and do not reflect the official policy or position of Mayo Clinic, Brooke Army Medical Center, the U.S. Army Medical Department, the U.S. Army Office of the Surgeon General, the Department of the Army, the Department of the Air Force, or the Department of Defense or the U.S. Government.

\section{Author details}

${ }^{1}$ Department of Otolaryngology-Head and Neck Surgery, Mayo Clinic, Rochester, MN 200 1st St SW55905, USA. ²Department of Otolaryngology-Head and Neck Surgery, San Antonio Uniformed Services Health Education Consortium, JBSA, Oddly, TX, USA. ${ }^{3}$ Department of Psychiatry and Psychology, Mayo Clinic, Rochester, MN, USA. ${ }^{4}$ Department of Quantitative Health Sciences, 
Mayo Clinic, Rochester, MN, USA. ${ }^{5}$ Department of Neurologic Surgery, Mayo Clinic, Rochester, MN, USA.

Received: 11 December 2021 Accepted: 22 January 2022

Published online: 29 January 2022

\section{References}

1. Tunkel DE, Bauer CA, Sun GH, et al. Clinical practice guideline: tinnitus. Otolaryngol Head Neck Surg. 2014;151:S1-40.

2. Henry JA, Reavis KM, Griest SE, et al. Tinnitus: an epidemiologic perspective. Otolaryngol Clin North Am. 2020:53:481-99.

3. Baguley D, McFerran D, Hall D. Tinnitus. Lancet. 2013;382:1600-7.

4. van Zon A, Smulders YE, Ramakers GG, et al. Effect of unilateral and simultaneous bilateral cochlear implantation on tinnitus: a prospective study. Laryngoscope. 2016;126:956-61.

5. Peter N, Liyanage N, Pfiffner F, Huber A, Kleinjung T. The influence of cochlear implantation on tinnitus in patients with single-sided deafness: a systematic review. Otolaryngol Head Neck Surg. 2019;161:576-88.

6. Holder JT, O'Connell B, Hedley-Williams A, Wanna G. Cochlear implantation for single-sided deafness and tinnitus suppression. Am J Otolaryngol. 2017;38:226-9.

7. Deep NL, Spitzer ER, Shapiro WH, Waltzman SB, Roland JT Jr, Friedmann DR. Cochlear implantation in adults with single-sided deafness: outcomes and device use. Otol Neurotol. 2021;42:414-23.

8. Mallen JR, Chiu J, Marquis $\mathrm{H}$, et al. Quantifying tinnitus suppression in cochlear implantation using tinnitus interval-limited tracking. Laryngoscope. 2020;130:2047-52.

9. Mertens $G$, De Bodt M, Van de Heyning P. Cochlear implantation as a long-term treatment for ipsilateral incapacitating tinnitus in subjects with unilateral hearing loss up to 10 years. Hear Res. 2016;331:1-6.

10. Newman CW, Jacobson GP, Spitzer JB. Development of the Tinnitus Handicap Inventory. Arch Otolaryngol Head Neck Surg. 1996;122:143-8.

11. Zeman F, Koller M, Figueiredo R, et al. Tinnitus Handicap Inventory for evaluating treatment effects: which changes are clinically relevant? Otolaryngol Head Neck Surg. 2011;145:282-7.

12. Meikle MB, Henry JA, Griest SE, et al. The Tinnitus Functional Index: development of a new clinical measure for chronic, intrusive tinnitus. Ear Hear. 2012;33:153-76.

13. Adamchic I, Langguth B, Hauptmann C, Tass PA. Psychometric evaluation of Visual Analog Scale for the assessment of chronic tinnitus. Am J Audiol. 2012;21:215-25.

14. Angara P, Tsang DC, Hoffer ME, Snapp HA. Self-perceived hearing status creates an unrealized barrier to hearing healthcare utilization. Laryngoscope. 2021;131:E289-95.

15. Limviriyakul S, Supavanich W. The validity and reliability of Tinnitus Handicap Inventory Thai version. J Med Assoc Thai. 2012;95:1433-40.

16. Barake R, Rizk SA, Ziade G, Zaytoun G, Bassim M. Adaptation of the Arabic Version of the Tinnitus Handicap Inventory. Otolaryngol Head Neck Surg. 2016;154:508-12.

17. Tobias CA, Llanes EG, Chiong C. Validity of a Filipino translation of the Tinnitus Handicap Inventory. Int Tinnitus J. 2012;17:64-9.

18. Monzani D, Genovese E, Marrara A, et al. Validity of the Italian adaptation of the Tinnitus Handicap Inventory; focus on quality of life and psychological distress in tinnitus-sufferers. Acta Otorhinolaryngol Ital. 2008:28:126-34

19. Zachariae R, Mirz F, Johansen LV, Andersen SE, Bjerring P, Pedersen CB. Reliability and validity of a Danish adaptation of the Tinnitus Handicap Inventory. Scand Audiol. 2000;29:37-43.

20. Engineer ND, Rosellini WM, Tyler RS. Willingness to accept and pay for implantable tinnitus treatments: a survey. Neuromodulation. 2013;16:154-62.

21. Roland JT Jr, Gantz BJ, Waltzman SB, Parkinson AJ. Long-term outcomes of cochlear implantation in patients with high-frequency hearing loss. Laryngoscope. 2018;128:1939-45.

22. Carlson ML. Cochlear implantation in adults. N Engl J Med. 2020;382:1531-42.

23. Leake PA, Snyder RL, Hradek GT, Rebscher SJ. Consequences of chronic extracochlear electrical stimulation in neonatally deafened cats. Hear Res. 1995;82:65-80.
24. Ni D, Shepherd RK, Seldon HL, Xu SA, Clark GM, Millard RE. Cochlear pathology following chronic electrical stimulation of the auditory nerve. I: Normal hearing kittens. Hear Res. 1992;62:63-81.

\section{Publisher's Note}

Springer Nature remains neutral with regard to jurisdictional claims in published maps and institutional affiliations.
Ready to submit your research? Choose BMC and benefit from:

- fast, convenient online submission

- thorough peer review by experienced researchers in your field

- rapid publication on acceptance

- support for research data, including large and complex data types

- gold Open Access which fosters wider collaboration and increased citations

- maximum visibility for your research: over 100M website views per year

At BMC, research is always in progress.

Learn more biomedcentral.com/submissions 